SCIENTIFIC REPORT

\title{
Choroidal expansion as a mechanism for acute primary angle closure: an investigation into the change of biometric parameters in the first 2 weeks
}

\author{
M Yang, T Aung, R Husain, Y-H Chan, L S Lim, S K L Seah, G Gazzard
}

Br J Ophthalmol 2005;89:288-290. doi: 10.1136/bjo.2004.048686

Background/aim: Choroidal expansion with anterior movement of the lens was recently proposed as a mechanism for acute primary angle closure (APAC). The aim of this study was to compare the biometric parameters, central anterior chamber depth, limbal chamber depth, lens thickness, and lens position, within 24 hours of presentation and 2 weeks later in eyes with APAC.

Methods: This was a prospective observational case series of 41 subjects with APAC. Subjects who presented with APAC were treated with medical therapy followed by laser iridotomy (LI) in both eyes once the acute attack was broken. Ocular biometry was performed in affected and fellow eyes before LI (baseline) and then 2 weeks later. Optical pachymetry was used to measure central anterior chamber depth (ACD) and the limbal chamber depth (LCD) was graded at the slit lamp. A-scan ultrasound was used to measure lens thickness (LT) and axial length (AL). Lens position (LP) was defined as $A C D+1 / 2 L T$.

Results: The majority of subjects were Chinese $(83 \%)$ and female (61\%), and the mean age was 60.4 (SD 10.3) years. In affected eyes, the ACD was $1.81(0.29) \mathrm{mm}$ before and $1.80(0.28) \mathrm{mm} 2$ weeks after $\mathrm{LI}(\mathrm{p}=0.63)$, while in fellow eyes, the ACD was $1.83(0.29) \mathrm{mm}$ and $1.81(0.38) \mathrm{mm}$, respectively $(p=0.21)$. There was no significant change in lens position, relative lens position, or axial length in both affected and fellow eyes over the 2 weeks.

Conclusions: There was no change observed in central anterior chamber depth, lens thickness, or lens position at the time of the acute attack compared to 2 weeks later in both APAC affected and fellow eyes. The findings do not support the hypothesis of lens movement due to choroidal expansion in APAC.

A cute primary angle closure (APAC) is a potentially blinding ocular condition. The incidence of APAC is especially high in east Asia, with Singaporeans having the highest reported incidence rate in the world of 12.2 per 100000 per year in those aged 30 years and older. ${ }^{1}$ Early biometric research by Lowe ${ }^{2}$ and Alsbirk, ${ }^{3}$ and confirmed by Friedman $e a^{4}{ }^{4}$ has shown that APAC eyes are smaller in axial length, have flatter corneas, shallower anterior chambers, and thicker lenses. Clearly, smaller ocular biometry is a risk factor for the condition. However, there could be other physiological factors which are equally important, especially with regard to triggering of the acute attack.

One mechanism proposed for APAC in anatomically predisposed eyes has been choroidal expansion causing a forward movement of the lens and greater iris convexity. ${ }^{5}$ Normally, an increase in volume posteriorly in the choroid would be accommodated by fluid exit from the anterior chamber without any shift in iris or lens position. However, the vitreous has a finite ability to transmit fluid because of its chemical structure. When transvitreous flow is insufficient to equalise the pressure differential, the result is anterior movement of the compressed vitreous humour, iris, and lens. With time, the vitreous compresses more, further decreasing its fluid conductivity and establishing a vicious cycle. In small eyes that are predisposed to PACG, forward lens movement of even a modest amount is believed to increase iris convexity and make acute angle closure more likely. ${ }^{5}$

The aim of this study was to investigate the evidence for choroidal expansion as a mechanism for APAC. Specifically we compared the biometric parameters, anterior chamber depth, lens thickness, and lens position within 24 hours of presentation and again after 2 weeks in eyes with APAC.

\section{METHODS}

This was a prospective observational case series of Asian subjects who presented with APAC. Written informed consent was obtained from all subjects and the study had the approval of the ethics committee of the Singapore National Eye Centre, and was performed according to the tenets of the Declaration of Helsinki. Demographic characteristics and ophthalmic data related to the APAC episode (such as laterality of affected eye, duration of acute symptoms and presenting IOP) were recorded for each subject. The following criteria were used to define cases of APAC:

(1) presence of at least two of the following symptoms: ocular or periocular pain, nausea and/or vomiting, an antecedent history of intermittent blurring of vision with haloes; and

(2) presenting intraocular pressure (IOP) of more than $28 \mathrm{~mm} \mathrm{Hg}$ (as measured by Goldmann applanation tonometry) and the presence of at least three of the following signs: conjunctival injection, corneal epithelial oedema, mid-dilated unreactive pupil, and shallow anterior chamber.

Upon presentation, medical therapy was instituted in order to first bring down the IOP. This comprised acetazolamide, given intravenously and orally, topical pilocarpine, timolol, and betamethasone. Once the acute attack was broken with reduction of intraocular pressure and improvement of corneal clarity, sequential laser iridotomy (LI) was performed in both eyes, usually within $24-48$ hours of presentation. There were

Abbreviations: $A C D$, anterior chamber depth; $A L$, axial length; $A P A C$, acute primary angle closure; LCD, limbal chamber depth; LI, laser iridotomy; LP, lens position; LT, lens thickness 


\begin{tabular}{ll}
$\begin{array}{l}\text { Table } 1 \\
\text { subjects }\end{array}$ & $\begin{array}{l}\text { Demographic characteristics of study } \\
\text { Nitute primary angle closure }\end{array}$ \\
\hline & Number $(\mathbf{n}=41)$ \\
\hline Sex & $16(39 \%)$ \\
Male & $25(61 \%)$ \\
Female & \\
Race & $34(83.0 \%)$ \\
Chinese & $5(12.2 \%)$ \\
Malay & $1(2.4 \%)$ \\
Others & $1(2.4 \%)$ \\
Age (years) & $60.4(10.3)$ \\
Mean (SD) & $35-99$ \\
Range & 60 \\
Median & \\
\hline
\end{tabular}

no cases that required a gonioplasty, pupilloplasty, or paracentesis.

There were two scheduled examinations: the first occurred within 24 hours of presentation and before LI (usually after medical therapy had successfully reduced IOP) and was defined as the baseline examination and the second occurred 2 weeks later. At each examination, the subjects' treatment, visual acuity, IOP, and anterior segment slit lamp findings were recorded. Central anterior chamber depth (ACD) and central corneal thickness were measured at the slit lamp by optical pachymetry (Depth Measuring Devices 1 and 2, HaagStreit, Bern, Switzerland). No correction was made for corneal radius or diameter, and cycloplegic agents were not used. "True" ACD (from the corneal endothelium to the anterior lens epithelium), calculated by subtracting the corneal thickness from the central ACD measurement, was used in the analysis. The limbal chamber depth (LCD) was also assessed using the Van Herick method and graded as a percentage fraction of the peripheral corneal thickness. ${ }^{6}$ After anaesthetising the eye to be examined with a drop of local anaesthetic, measurement of the lens thickness (LT) and axial length (AXL) of the eyeball were performed by A-mode applanation ultrasonography (Sonomed A2500, Haag-Streit, Koniz, Switzerland). Special care was taken in aligning the transducer beam probe along the optical axis and to exert minimal corneal pressure by mounting the probe on a tonometer mount set. Measurements were performed until five consecutive readings with a standard deviation of $\leqslant 0.05$ were obtained for each variable. Lens position (LP) was defined as ACD $+1 / 2$ LT and relative lens position (RLP) was defined as LP/AL. All measurements were performed on subjects in the study by one of two observers (the same observer for each subject), who were masked to previous examinations.

In the interim period between study visits, APAC subjects were managed by their respective physicians and flexibility was permitted in the treatment. Those who underwent cataract or filtering surgery during the study period were excluded from the analysis as intraocular surgery may affect the ACD. The need and timing for surgery was made at the discretion of the surgeon, and there was no specified indication for surgery in the study protocol. Subjects with secondary angle closure, such as lens induced glaucoma, neovascular glaucoma, or uveitis were also excluded.

\section{RESULTS}

A total of 41 Asian subjects completed this study. The majority of them were Chinese (83\%) and female $(61 \%)$ and the mean age was 60.4 (SD 10.3) years (table 1). Disease presentation was unilateral in all cases.

Table 2 shows the ocular biometric parameters in affected and fellow eyes measured before LI and then 2 weeks later. In affected eyes, the ACD was 1.81 (SD 0.29) $\mathrm{mm}$ before (baseline) and $1.80(0.28) \mathrm{mm} 2$ weeks after LI $(\mathrm{p}=0.63)$, while in fellow eyes, the ACD was $1.83(0.29) \mathrm{mm}$ and 1.81 (0.38) $\mathrm{mm}$ respectively $(\mathrm{p}=0.21)$.

There was also no significant change in AXL, LT, LP, or RLP in affected and fellow eyes over the 2 weeks. The LCD in affected eyes was 13.1 (2.7) percentage corneal thickness) at baseline and $25.4(4.2)$ at 2 weeks after 2 weeks $(p=0.006)$, while in fellow eyes, the LCD was 13.9 (2.2) and 23.8 (3.1), respectively $(\mathrm{p}=0.001)$.

\section{DISCUSSION}

The purpose of this study was to review the hypothesis of anterior lens displacement secondary to choroidal expansion as a possible mechanism for APAC. In initial preliminary investigations, it had been suggested that LI was associated with a change in central ACD (Gazzard, personal communication quoted in Quigley et $\left.a l^{5}\right)$. However, after complete analysis of the data, our results did not demonstrate any evidence of lens movement in patients presenting with APAC as there was no significant change in ACD, LP, or RLP when compared before LI and 2 weeks later.

\begin{tabular}{|c|c|c|c|c|}
\hline Ocular parameter (mean (SD)) & Affected eye & $\begin{array}{l}\text { p Value (week } 2 \\
\text { v baseline) }\end{array}$ & Fellow eye & $\begin{array}{l}\mathrm{p} \text { Value (week } 2 \\
\mathrm{v} \text { baseline) }\end{array}$ \\
\hline \multicolumn{5}{|l|}{ Anterior chamber depth $(\mathrm{ACD})$} \\
\hline Baseline & $1.81(0.29)$ & & $1.83(0.29)$ & \\
\hline Week 2 & $1.80(0.28)$ & $0.63^{*}$ & $1.81(0.38)$ & $0.21 \dagger$ \\
\hline \multicolumn{5}{|l|}{ Axial length (AXL) } \\
\hline Baseline & $21.92(0.82)$ & & $21.93(0.82)$ & \\
\hline $\begin{array}{l}\text { Week } 2 \\
\text { Lens thickness (LT) }\end{array}$ & $21.99(0.86)$ & $0.52^{*}$ & $21.86(0.83)$ & $0.90 \dagger$ \\
\hline Baseline & $5.17(0.66)$ & & $5.13(0.50)$ & \\
\hline Week 2 & $5.02(0.67)$ & $0.09^{*}$ & $5.06(0.60)$ & $0.66 \dagger$ \\
\hline \multicolumn{5}{|l|}{ Lens position (ACD+LT/2) } \\
\hline Baseline & $4.39(0.43)$ & & $4.40(0.35)$ & \\
\hline Week 2 & $4.31(0.46)$ & $0.24^{*}$ & $4.34(0.51)$ & $0.81 \dagger$ \\
\hline \multicolumn{5}{|l|}{ Relative lens position (LP/AXL) } \\
\hline Baseline & $0.20(0.02)$ & & $0.20(0.02)$ & \\
\hline Week 2 & $0.19(0.02)$ & $0.16^{*}$ & $0.19(0.02)$ & $0.86 \dagger$ \\
\hline \multicolumn{5}{|l|}{ Limbal chamber depth (LCD) } \\
\hline Baseline & $13.1(2.7)$ & & $13.9(2.2)$ & \\
\hline Week 2 & $25.4(4.2)$ & $0.006^{*}$ & $23.8(3.1)$ & $0.001 \dagger$ \\
\hline
\end{tabular}


Our findings are in agreement with previous studies that measured central anterior chamber depth before and after an iridotomy. ${ }^{7-10}$ No significant deepening was found, though the peripheral anterior chamber depth was found to increase and eyes had a flatter peripheral iris configuration after iridotomy. ${ }^{2811} 12$ The main criticism of these previous studies was that only a subset of the patients had APAC on presentation and, hence, the conclusions may not be representative of this body of patients as a whole.

There are certain limitations within this study that should be highlighted. Firstly, measurements were made after beginning medical therapy for the acute attack and so potential trigger factors, which lead to an initial anterior movement of a compressed vitreous humour, iris, and lens, may already have resolved. Hence, the ACD measured just before the LI may only be an approximation of the true baseline ACD at the onset of an attack. This highlights the difficulty in identifying subtle physiological changes in such a dynamic situation. Medical therapy such as acetazolamide, pilocarpine, and steroids, as well as the laser iridotomy itself could have an effect on possible choroidal expansion and/or lens displacement, and may even mask small changes in these forces. Investigations like B-scan ultrasonography and ultrasound biomicroscopy which may be useful in directly evaluating the choroidal thickness were unfortunately not performed in this study. Finally, our sample of 41 eyes may be too small to detect small effects of the variables that were evaluated.

In addition, choroidal expansion (oedema) is also frequently seen in clinical states of low as well as high intraocular pressures. Hypotony is associated with the breakdown of blood-ocular barriers and choroidal expansion can take place with extravasation of blood proteins followed by tissue retention of water. Subsequently, choroidal detachment can take place with accumulation of fluid in the suprachoroidal space. Gazzard et $a l^{13}$ reported three patients in whom suprachoroidal fluid was present after resolution of the acute attack. He concluded that APAC may be complicated by secondary suprachoroidal fluid collections probably as a result of aggressive hypotensive therapy but it cannot be excluded that the suprachoroidal fluid existed before the acute attack. It may well be that choroidal expansion initially precipitated the attack and persisted even after it was broken, since choroidal effusion can exist in both states of high and low intraocular pressures. ${ }^{14}$ If this fluid persisted at the time of second measurement then there would be no net change in lens position between measurements.

In summary, although the proposed mechanism of choroidal expansion as a physiological trigger accounting for APAC in anatomically predisposed eyes is biologically plausible, we did not find any evidence supporting this particular mechanism in this series.

\section{Authors' affiliations}

M Yang, T Aung, R Husain, L S Lim, S K L Seah, G Gazzard, Singapore National Eye Centre, Singapore

T Aung, Y-H Chan, National University of Singapore, Singapore

R Husain, G Gazzard, Institute of Ophthalmology, London, UK

Financial support: Nil.

Commercial interest: Nil.

Correspondence to: Dr Tin Aung, Glaucoma Department, Singapore National Eye Centre, 11 Third Hospital Avenue, Singapore 168751. tin11@pacific.net.sg

Accepted for publication 1 August 2004

\section{REFERENCES}

1 Seah SKL, Foster PJ, Chew PTK, et al. Incidence of acute primary angle closure glaucoma in Singapore. An island-wide survey. Arch Ophthalmol 1997; 115:1436-40.

2 Lowe RF. Aetiology of the anatomical basis for primary angle-closure glaucoma. Br J Ophthalmol 1970;54:161-9.

3 Alsbirk PH. Primary angle-closure glaucoma: oculometry, epidemiology, and genetics in a high risk population. Acta Ophthalmol 1976;54:5-31.

4 Friedman DS, Gazzard G, Foster P, et al. Ultrasound biomicroscopy, Scheimpflug photography, and novel provocative tests in contralateral eyes of Chinese patients presenting with acute angle-closure. Arch Ophthalmol 2003;121:633-42.

5 Quigley HA, Friedman DS, Congdon NG. Possible mechanisms of primary angle-closure and malignant glaucoma. J Glaucoma 2003;12:167-80.

6 Foster P, Devereux J, Alsbirk PH, et al. Detection of gonioscopically occludable angles and primary angle closure glaucoma by estimation of limbal chamber depth in Asians: modified grading scheme. Br J Ophthalmol 2000;84:186-92

7 Lee DA, Brubaker RF, Ilstrup DM. Anterior chamber dimensions in patients with narrow angles and angle-closure glaucoma. Arch Ophthalmol 1984; 102:46-50

8 Jin JC, Anderson DR. The effect of iridotomy on iris contour. Am J Ophthalmol 1990;1 10:260-3.

9 Marraffa M, Marchini G, Pagliarusco A, et al. Ultrasound biomicroscopy and corneal endothelium in Nd:YAG-laser iridotomy. Ophthalmic Surg Lasers 1995;26:519-23.

10 Jacobs IH, Krohn DL. Central anterior chamber depth after laser iridotomy. Am J Ophthalmol 1980;89:865-7.

11 Gazzard G, Friedman DS, Devereux JG, et al. A prospective ultrasound biomicroscopy evaluation of changes in anterior segment morphology after laser iridotomy in Asian eyes. Ophthalmology 2003; 1 10:630-8.

12 Caronia RM, Liebmann JM, Stegman Z, et al. Increase in iris-lens contact after laser iridotomy for pupillary block angle closure. Am J Ophthalmol 1996; 122:53-7.

13 Gazzard G, Friedman DS, Devereux J, et al. Primary angle closure glaucoma associated with supra-choroidal fluid in three Chinese patients. Eye $2001 ; 15: 358-60$

14 Liebmann JM, Weinreb RN, Ritch R. Angle-closure glaucoma associated with occult annular ciliary body detachment. Arch Ophthalmol 1998;116:731-5. 